Article

\title{
Optimal Timing to Trade along a Randomized Brownian Bridge
}

\author{
Tim Leung ${ }^{1, *}$ (D), Jiao $\mathrm{Li}^{2}$ and ${\mathrm{Xin} \mathrm{Li}^{3}}^{3}$ \\ 1 Department of Applied Mathematics, University of Washington, Seattle, WA 98195, USA \\ 2 APAM Department, Columbia University, New York, NY 10027, USA; j14170@columbia.edu \\ 3 Bank of America Merrill Lynch, One Bryant Park, New York, NY 10036, USA; xinli.columbia@gmail.com \\ * Correspondence: timleung@uw.edu
}

Received: 31 December 2017; Accepted: 3 August 2018; Published: 30 August 2018

check for updates

\begin{abstract}
This paper studies an optimal trading problem that incorporates the trader's market view on the terminal asset price distribution and uninformative noise embedded in the asset price dynamics. We model the underlying asset price evolution by an exponential randomized Brownian bridge $(\mathrm{rBb})$ and consider various prior distributions for the random endpoint. We solve for the optimal strategies to sell a stock, call, or put, and analyze the associated delayed liquidation premia. We solve for the optimal trading strategies numerically and compare them across different prior beliefs. Among our results, we find that disconnected continuation/exercise regions arise when the trader prescribe a two-point discrete distribution and double exponential distribution.
\end{abstract}

Keywords: speculative trading; Brownian bridge; optimal stopping; variational inequality

MSC: 60G40; 62L15; 91G20; 91G80

JEL Classification: C41; G11; G13

\section{Introduction}

One fundamental problem faced by all traders is to determine when to sell an asset or financial derivative over a given trading horizon. The optimal trading decision depends crucially on the trader's subjective belief of the distribution of the asset price in the future and the observed price fluctuations. By monitoring the asset price evolution over time, the trader decides whether to sell the asset or derivative at the prevailing market price, or continue to wait until a later time. In this paper, we tackle this problem by constructing models that reflect the two major features: (i) the trader's market view on the terminal asset price distribution and the uninformative noise embedded in the asset price dynamics, and (ii) the timing option that gives rise to an optimal stopping problem corresponding to the trader's asset or derivative liquidation.

In order to describe the asset price evolution, we present a randomized Brownian bridge $(\mathrm{rBb})$ model, whereby the log-price of the asset follows a Brownian bridge with a randomized endpoint representing the random terminal log-price. In turn, the trader's prior belief and learning mechanism can be encoded in the drift of the log-price process. Our model is adapted from the novel work by Cartea et al. (2016).

They model the asset's mid-price as a randomized Brownian bridge, and solve a trading problem that maximizes the expected trading revenue by placing either limit or market orders while penalizing the running inventory. In comparison, the underlying asset price in our model is an exponential $\mathrm{rBb}$ with different randomized endpoints, with examples including discrete, normal, and double exponential distribution. In addition, we introduce an optimal stopping approach to the trader's 
liquidation problem, which is applicable not only to selling the underlying asset, but also options written on it.

To solve the trader's optimal stopping problem, we devise and apply a number of analytical tools. We first define the optimal liquidation premium that represents the additional value from optimally waiting to sell, as opposed to immediate liquidation. Indeed, as soon as this premium vanishes, it is optimal for the trader to sell. On the other hand, if this premium is always strictly positive, then the trader finds it optimal to wait through maturity. Therefore, the optimal liquidation premium provides not only new financial interpretations, but also another avenue of analytical investigation of the optimal stopping problem. Among our results, we identify the conditions under which it is optimal to immediately liquidate, or hold the asset/option position through expiration. Furthermore, we prove that the optimal strategy to liquidate a long-call-short-put position is identical to the optimal strategy to sell the underlying stock under the same trading horizon. This timing parity holds for any distribution of the randomized endpoint. Moreover, we derive the variational inequality associated with the optimal stopping problem, and present a finite-difference method to solve for the optimal trading boundaries.

In the literature, Brownian bridges have been used to represent market uncertainty or uninformative noise (see e.g., Brody et al. (2009), Hughston and Macrina (2012), and Macrina (2014)). The randomized Brownian bridge model in this paper belongs to the information-based approach to pricing and trading. Among related studies, Brody et al. (2008) and Filipović et al. (2012) study information-based models where the asset prices are computed via conditional expectation with respect to an information process.

Randomized Brownian bridges, or their variations, have great potential applicability in a number of finanical applications. For example, while futures prices are supposed to be equal to the spot price in theory, it is observed that some commodity futures prices do not exactly converge to the corresponding spot prices upon maturity (see Guo and Leung (2017)). Brennan and Schwartz (1990) and Dai et al. (2011) investigate arbitrage strategies on stock index futures where the index arbitrage basis is assumed to follow a Brownian Bridge. One can also look for more potential applications in index tracking and exchange-traded funds (see Leung and Santoli (2016)). The randomized endpoint provides added flexibility in modeling random shocks to the asset price on a future date, which is applicable to events such as Federal Reserve announcements, and earnings surprises (see e.g., Johannes and Dubinsky (2006) and Leung and Santoli (2014)).

As for optimal stopping problems involving Brownian bridges, Ekström and Wanntorp (2009) consider optimal single stopping of a Brownian bridge or odd powers of a Brownian bridge, without discounting. Ekström and Vaicenavicius (2017) use an optimal stopping approach to maximize the expected value of a Brownian bridge with an unknown pinning point. The optimal double problem is further studied in Baurdoux et al. (2015), where they maximize the expected spread between the payoffs at the entry and exit times where the underlying follows a Brownian bridge.

In related works on optimal stopping problems for securities trading under a finite horizon setting, Leung and Ludkovski $(2011,2012)$ introduce the concept of delayed purchase premium and analyze the problem of purchasing equity European and American options in an incomplete market, where the investor's belief on asset price dynamics may differ from prevalent market views. Leung and Liu (2012) analyze the delayed purchase premium associated with credit derivatives under a multi-factor intensity-based default risk framework, and derive the optimal trading strategies. In Leung and Shirai (2015), the authors study the optimal timing to sell an asset or option subject to a path-dependent risk penalty.

The rest of the paper is structured as follows. In Section 2, we present the randomized Brownian bridge model for the underlying asset price, show how different prior beliefs are encapsulated in the stochastic differential equation corresponding to the price dynamics, and illustrate the price behaviors through Monte Carlo simulation. In Section 3, we formulate and analyze the optimal liquidation problem and present numerical results to illustrate the optimal trading strategies. In Section 4, 
we summarize the numerical algorithm used for solving the optimal stopping problems under different settings in this paper. A number of proofs are collected in Section 6.

\section{Prior Belief and Price Dynamics}

The model consists of a single asset whose positive price process is denoted by $\left(S_{t}\right)_{0 \leq t \leq T}$. The trader in our model specifies a prior belief on the future distribution of the stock price at a fixed future time $T$. We denote $X_{t}=\log S_{t}$ to be the log-price of the asset, with $X_{0}$ being the stock's initial log-price. The trader's belief is described by a real-valued random variable $D$ to be realized at time $T$ so that the terminal log-price is given by

$$
X_{T}=X_{0}+D
$$

To avoid arbitrage, we require that $D$ have finite second moment and $\mathbb{P}(D>r T) \in(0,1)$, where $r$ is positive risk-free interest rate, under the historical probability measure $\mathbb{P}$.

As time progresses, new information arrives in the form of changes in asset price. The price fluctuation can also be viewed as noise prior to the realization of the terminal log-price $D$. We model the log-price process as a randomized Brownian bridge. To this end, we first let $\left(\beta_{t}\right)_{0 \leq t \leq T}$ be a standard Brownian bridge

$$
\beta_{t}=B_{t}-\frac{t}{T} B_{T}, \quad t \in[0, T]
$$

where $\left(B_{t}\right)_{0 \leq t \leq T}$ is a standard Brownian motion. The process $\beta$ starts at 0 and ends at 0 . It can be viewed as uninformative noise, which vanishes both at times 0 and $T$. We assume that the processes $B$ and thus $\beta$ are independent of the random variable $D$.

Then, the log-price process is given by

$$
X_{t}=X_{0}+\sigma \beta_{t}+\frac{t}{T} D, \quad t \in[0, T]
$$

where $\sigma>0$ is a constant parameter. The trader observes the price evolution of the asset, and the corresponding filtration $\mathbb{F} \equiv\left(\mathcal{F}_{t}\right)_{0 \leq t \leq T}$ is generated by the log-price process $X$. In other words, the trader does not directly observe the standard Brownian bridge $\beta$ over time. Mathematically, this means that $\beta_{t}$ is not adapted to $\mathbb{F}$. The financial interpretation is that $\beta$ reflects the uninformative noise in the markets such as market sentiments and rumors. Without direct observation of $\beta_{t}$, the trader is unable to decompose $X_{t}$ into $\beta_{t}$ and $D$ at any time $t<T$. At time $T$, the trader observes the realization of $D$ and thus $X_{T}$. The parameter $\sigma$ allows us to control effect of $\beta$ on the log-price fluctuation. By inspecting Equation (3), the ratio $\frac{t}{T}$ can be viewed as the rate at which the value of $D$ is revealed, going linearly from fully hidden at time 0 to fully revealed at time $T$.

By Proposition 1 of Cartea et al. (2016), the log-price process satisfies the stochastic differential equation (SDE)

$$
d X_{t}=A\left(t, X_{t}\right) d t+\sigma d W_{t}
$$

for $t \in[0, T]$, where $\left(W_{t}\right)_{0 \leq t \leq T}$ is an $\mathbb{F}$-adapted standard Brownian motion under the probability measure $\mathbb{P}$. The drift term is

$$
A\left(t, X_{t}\right)=\frac{a\left(t, X_{t}\right)-\left(X_{t}-X_{0}\right)}{T-t}
$$


where

$$
a(t, x):=\mathbb{E}\left[D \mid X_{t}=x\right]=\frac{\int_{-\infty}^{\infty} z \exp \left(z \frac{x-X_{0}}{\sigma^{2}(T-t)}-z^{2} \frac{t}{2 T \sigma^{2}(T-t)}\right) d F(z)}{\int_{-\infty}^{\infty} \exp \left(z \frac{x-X_{0}}{\sigma^{2}(T-t)}-z^{2} \frac{t}{2 T \sigma^{2}(T-t)}\right) d F(z)}
$$

and $F(\cdot)$ is the cumulative distribution function of the random variable $D$. We refer to Appendix A of Cartea et al. (2016) for the derivation of Equation (4). The $\mathbb{F}$-Brownian motion $W_{t}$ appearing in (4) can be considered as the market information accessible by the trader. Unlike $\beta_{t}$, the value of $W_{t}$ contains real information relevant to the risky asset price. Equations (4)-(6) represent how the price innovations reflect the probability distribution of $D$ and market information flow. The incomplete information in (3) is reformulated as a complete information by projecting the log-price innovations onto the observable filtration. In that sense, the information-based approach is more flexible to add additional interpretation and intuition based on observable price process, as we present in the following sessions of the paper.

To understand the conditional expectation in (6), we start with the definition

$$
\mathbb{E}\left[D \mid X_{t}=x\right]=\int_{-\infty}^{\infty} z \pi_{t}(z) d z,
$$

where $\pi_{t}(z)$ is the conditional probability density or mass function for the random variable $D$ defined by

$$
\pi_{t}(z)=\frac{d}{d z} \mathbb{P}\left(D \leq z \mid X_{t}=x\right) .
$$

Using Bayes formula, the conditional probability density is given by

$$
\pi_{t}(z)=\frac{p(z) \rho_{X_{t}}(x \mid D=z)}{\int_{-\infty}^{\infty} p(z) \rho_{X_{t}}(x \mid D=z) d z}
$$

where $p(z)$ denotes the probability density or mass function for $D$, and $\rho_{X_{t}}(x \mid D=z)$ denotes the conditional density function for the random variable $X_{t}$ given $D=z$. According to (3), for any fixed $t \in[0, T), X_{t}$ given $D=z$ is Gaussian, i.e.,

$$
\left.X_{t}\right|_{D=z} \sim \mathcal{N}\left(X_{0}+\frac{t}{T} z, \sigma^{2} \frac{t}{T}(T-t)\right) .
$$

Therefore, we can express the conditional probability density for $X_{t}$ as

$$
\rho_{X_{t}}(x \mid D=z)=\frac{1}{\sigma \sqrt{2 \pi \frac{t(T-t)}{T}}} \exp \left(-\frac{T\left(x-X_{0}-\frac{t}{T} z\right)^{2}}{2 \sigma^{2} t(T-t)}\right) .
$$

Substituting this expression into (8), we have

$$
\pi_{t}(z)=\frac{p(z) \exp \left(z \frac{x-X_{0}}{\sigma^{2}(T-t)}-z^{2} \frac{t}{2 T \sigma^{2}(T-t)}\right)}{\int_{-\infty}^{\infty} p(z) \exp \left(z \frac{x-X_{0}}{\sigma^{2}(T-t)}-z^{2} \frac{t}{2 T \sigma^{2}(T-t)}\right) d z} .
$$

Finally, Equation (6) follows after we substitute (9) into (7).

The asset price is an exponential $\mathrm{rBb}$ defined by $S_{t}=\exp \left(X_{t}\right)$. By Ito's lemma, we obtain the SDE

$$
d S_{t}=\left(A\left(t, X_{t}\right)+\frac{\sigma^{2}}{2}\right) S_{t} d t+\sigma S_{t} d W_{t}, \quad 0 \leq t \leq T
$$


In this paper, we will consider three different distributions for $D$ : two-point discrete distribution, normal distribution, and double exponential distribution. The first one is discrete while the other two are continuous distributions. Compared to the normal distribution, the double exponential distribution is capable of generating two heavier tails that can be symmetric or asymmetric. Next, let us illustrate the effect of the distribution of $D$ on the asset price dynamics.

Example 1. Suppose that the trader's prior belief on the future log-price follows a two-point discrete distribution defined by

$$
D_{\delta}=\left\{\begin{array}{l}
\delta_{u} \text { with probability } p_{u} \\
\delta_{d} \text { with probability } p_{d}=1-p_{u}
\end{array}\right.
$$

with mean and variance, respectively,

$$
\begin{aligned}
\mathbb{E}\left[D_{\delta}\right] & =\delta_{u} p_{u}+\delta_{d} p_{d} \\
\operatorname{Var}\left[D_{\delta}\right] & =\delta_{u}^{2} p_{u}\left(1-p_{u}\right)+\delta_{d}^{2} p_{d}\left(1-p_{d}\right)-2 \delta_{u} \delta_{d} p_{u} p_{d} .
\end{aligned}
$$

Then, the drift term $A(t, x)$ of $X_{t}$ in (4) is given by

$$
A(t, x)=\frac{1}{T-t}\left[\frac{\delta_{u} u(t, x)+\delta_{d} d(t, x)}{u(t, x)+d(t, x)}-\left(x-X_{0}\right)\right]
$$

where

$$
\begin{aligned}
& u(t, x)=p_{u} \exp \left(\delta_{u} \frac{x-X_{0}}{\sigma^{2}(T-t)}-\delta_{u}^{2} \frac{t}{2 T \sigma^{2}(T-t)}\right), \\
& d(t, x)=p_{d} \exp \left(\delta_{d} \frac{x-X_{0}}{\sigma^{2}(T-t)}-\delta_{d}^{2} \frac{t}{2 T \sigma^{2}(T-t)}\right) .
\end{aligned}
$$

A special case of Example 1 is when $D$ is a constant $\delta$. Then, the log-price process (4) is simply a Brownian bridge that starts at $X_{0}$ and ends at $X_{0}+\delta$. Its SDE is given by

$$
d X_{t}=\frac{X_{0}+\delta-X_{t}}{T-t} d t+\sigma d W_{t}, \quad 0 \leq t \leq T
$$

Example 2. Suppose that the trader's prior belief on the future log-price follows a normal distribution with mean $\mu$ and variance $\sigma_{D}^{2}$, i.e.,

$$
D_{n} \sim \mathcal{N}\left(\mu, \sigma_{D}^{2}\right)
$$

Note that $\sigma_{D}$ is different from $\sigma$ in (4). The drift of $X_{t}$ in (4) is given by

$$
A(t, x)=\frac{\left(\sigma_{D}^{2}-T \sigma^{2}\right)\left(x-X_{0}\right)+\mu \sigma^{2} T}{t \sigma_{D}^{2}+T \sigma^{2}(T-t)} .
$$

The derivation is presented in Section 6.1.

As an alternative continuous distribution to the normal distribution, we now consider the double exponential distribution, which has been used to model random jumps in asset prices (see e.g., Kou (2002)).

Example 3. Suppose that the trader's prior belief on the future log-price is represented by a double exponential random variable $D_{e}$ with the $p d f$ 


$$
f(z)=\mathbf{1}_{\{z<\theta\}} p_{1} \lambda_{1} e^{\lambda_{1}(z-\theta)}+\mathbf{1}_{\{z \geq \theta\}} p_{2} \lambda_{2} e^{-\lambda_{2}(z-\theta)},
$$

where $p_{1}, p_{2}>0$ and $p_{2}=1-p_{1}$. The mean and variance of $D_{e}$ are, respectively,

$$
\begin{aligned}
\mathbb{E}\left[D_{e}\right] & =\theta-\frac{p_{1}}{\lambda_{1}}+\frac{p_{2}}{\lambda_{2}} \\
\operatorname{Var}\left[D_{e}\right] & =\frac{2 p_{1}}{\lambda_{1}^{2}}+\frac{2 p_{2}}{\lambda_{2}^{2}}-\left(\frac{p_{1}}{\lambda_{1}}-\frac{p_{2}}{\lambda_{2}}\right)^{2} .
\end{aligned}
$$

The drift of $X_{t}$ in (4) is given by

$$
A(t, x)=\frac{1}{T-t}\left[\frac{\sum_{i=1,2} N_{i}(t, x)}{\sum_{i=1,2} H_{i}(t, x)}-\left(x-X_{0}\right)\right],
$$

where

$$
\left\{\begin{aligned}
N_{i}(t, x)= & (-1)^{i} p_{i} \lambda_{i} \frac{1}{2 \zeta} \exp \left(-\zeta\left(\theta+\frac{b_{i}}{2 \zeta}\right)^{2}\right) \exp \left(\frac{b_{i}^{2}-4 \zeta c_{i}}{4 \zeta}\right) \\
& -p_{i} \lambda_{i} \frac{b_{i}}{2 \zeta} \frac{\sqrt{\pi}}{\sqrt{\zeta}} \exp \left(\frac{b_{i}^{2}-4 \zeta c_{i}}{4 \zeta}\right) \Phi\left((-1)^{i-1} d_{i}\right) \\
H_{i}(t, x)= & p_{i} \lambda_{i} \frac{\sqrt{\pi}}{\sqrt{\zeta}} \exp \left(\frac{b_{i}^{2}-4 \zeta c_{i}}{4 \zeta}\right) \Phi\left((-1)^{i-1} d_{i}\right)
\end{aligned}\right.
$$

and

$$
\left\{\begin{array}{l}
\zeta \equiv \zeta(t)=\frac{t}{2 T \sigma^{2}(T-t)} \\
b_{i} \equiv b_{i}(t, x)=-\left(\frac{x-X_{0}}{\sigma^{2}(T-t)}+(-1)^{i-1} \lambda_{i}\right) \\
c_{i}=(-1)^{i-1} \lambda_{i} \theta \\
d_{i} \equiv d_{i}(t, x)=\sqrt{2 \zeta(t)}\left(\theta+\frac{b_{i}(t, x)}{2 \zeta(t)}\right)
\end{array}\right.
$$

and $\Phi(\cdot)$ is the standard normal cumulative distribution function. We present the computations in Section 6.2.

The paths of the log-price process $X$ can be simulated over discrete times using the standard Euler-Maruyama method. Denote $\delta t$ as the discretization step, and set $t_{i}=i \delta t$, for $i=0,1,2, \ldots$ Then, the path of $X$, starting at $X_{0}$ at time 0 , can be simulated iteratively as follows:

$$
X_{t_{i+1}}=X_{t_{i}}+A\left(t_{i}, X_{t_{i}}\right) \delta t+\sigma \sqrt{\delta t} \epsilon_{i}
$$

where $\left(\epsilon_{i}\right)_{i=1,2, \ldots}$ is a sequence of independent and identically distributed $\mathcal{N}(0,1)$ random variables. This method does not require the simulation of the random variable $D$ directly because the distributional characteristics of $D$ are encapsulated in the drift function $A(t, x)$ (see (14), (19) and (23)). The procedure takes in the current value of $X_{t_{i}}$ at each time step to compute the drift $A\left(t_{i}, X_{t_{i}}\right)$, and each path evolves without the knowledge of the Brownian bridge $\beta$ or the realization of the random variable $D$ as both $\beta$ and $D$ are never simulated. This is consistent with the fact that the trader cannot observe $D$ prior to $T$, and only has knowledge of the log-price process $X$ over time but not the Brownian bridge $\beta$. The paths will end up with the terminal distribution resembling that 
of $D$. In Example 1, under two-point discrete distribution for $D$, simulating according to (24) will generate a path ending up at either $X_{0}+\delta_{u}$ or $X_{0}+\delta_{d}$, each with probability $p_{u}$ and $p_{d}$, respectively. For instance, in Figure 1a, we have $p_{1}=p_{2}=0.5$, meaning that about half of the paths will end up at either $X_{0}+\delta_{u}$ or $X_{0}+\delta_{d}$.

As seen in Examples 1-3, the specification of the terminal log-price distribution directly affects the drift of $X_{t}$. To better observe the different structures of the drift $A(t, x)$ under different distributions for $D$, we plot in Figure 2 the function $A(t, x)$ at $t=0.1$ (panel (a)) and $t=0.8$ (panel (b)) with the three distributions sharing the same mean and variance. Under the normal distribution, $A(t, x)$ is linear in $x$. In contrast, it is neither linear nor monotone under the two-point discrete and double exponential distributions. Moreover, under the two-point distribution, $A$ is positive when the stock price is low and negative when the stock price is relatively high, meaning that the asset price tends to have positive drift when prices are low and negative drift when prices are high. However, the opposite is observed in $A$ under the normal and double exponential distributions.

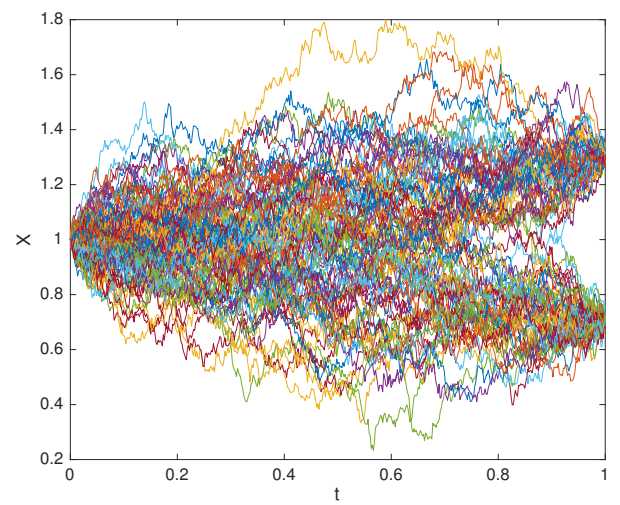

(a)

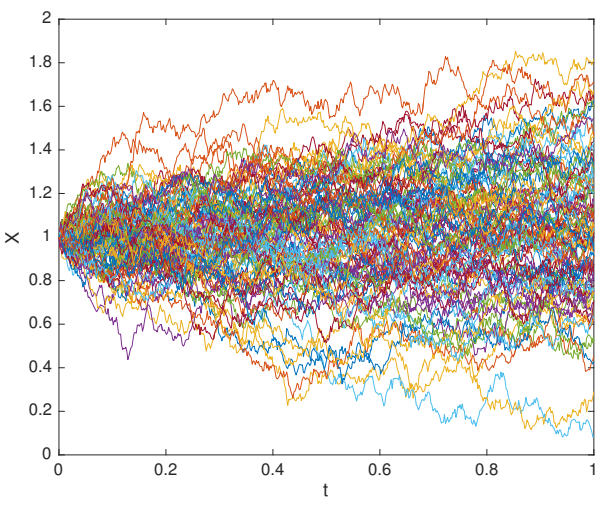

(b)

Figure 1. Path simulation of (4) with the prior belief on the future log-price following (a) two-point discrete distribution and (b) normal distribution. Parameters: (a) $\delta_{u}=0.3, \delta_{d}=-0.3, p_{u}=0.5, p_{d}=0.5$; (b) $\mu=0, \sigma_{D}=0.3$. Common parameters: $X_{0}=1, T=1, \sigma=0.4$.

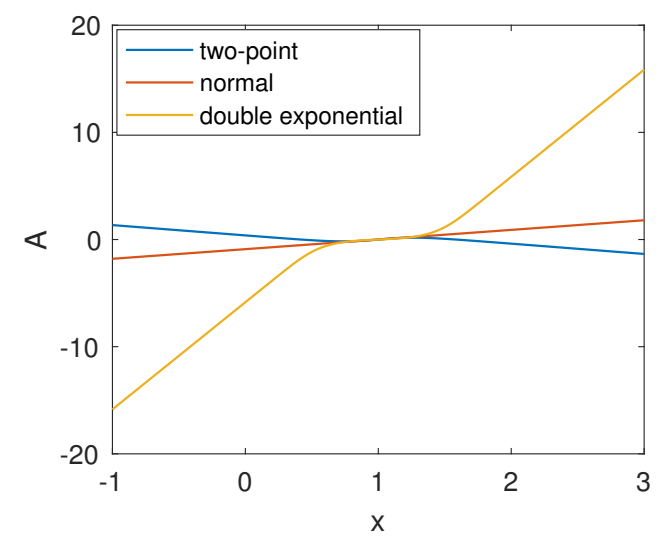

(a)

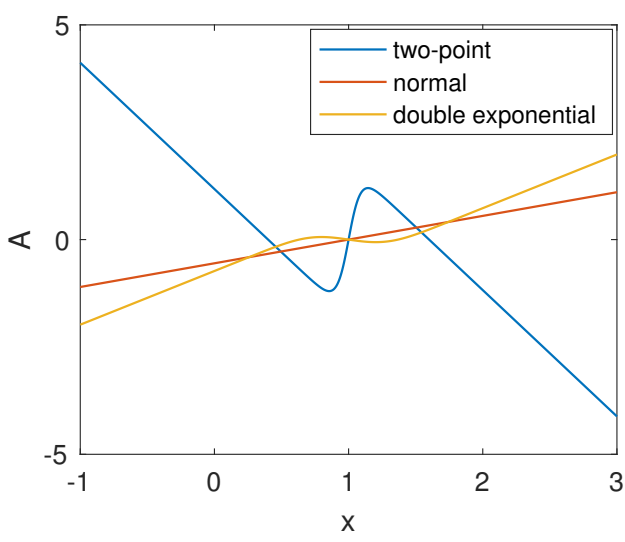

(b)

Figure 2. $A(t, x)$ under three different distribution with common MEAN $=0$ and VAR $=0.36$ at $t=0.1$ (a) and $t=0.8$ (b). Parameters: (two-point) $\delta_{u}=-\delta_{d}=0.6, p_{u}=p_{d}=0.5$; (normal) $\mu=0, \sigma_{D}=0.6$; (double exponential) $\theta=0, p_{1}=p_{2}=0.5, \lambda_{1}=\lambda_{2}=2.357$. Common parameters: $S_{0}=2.72\left(X_{0}=1\right), r=0.1, \bar{T}=1, T=1.1, \sigma=0.4$. 


\section{Optimal Liquidation Problems}

With the asset price dynamics given above, we now consider a trader who holds the underlying asset $S$ or an option written on $S$, and seeks to maximize the expected value from selling the security. Let $f(t, x) \in C^{2}([0, \infty) \times \mathbb{R})$ be a generic reward function, representing the value received from the security sale at time $t$ at log-price $x$. We assume a constant interest rate $r>0$, which is also the discount rate used by the trader.

In order to determine the optimal timing to sell, the trader solves the optimal stopping problem

$$
V(t, x)=\sup _{\tau \in \mathcal{T}_{t, \bar{T}}} \mathbb{E}\left\{e^{-r(\tau-t)} f\left(\tau, X_{\tau}\right) \mid X_{t}=x\right\},
$$

where $\mathcal{T}_{t, \bar{T}}$ is the set of all stopping times with respect to $\mathbb{F}$ taking values between $t$ and $\bar{T}$, with $0 \leq t \leq \bar{T} \leq T$. Here, $\bar{T}$ is the trading deadline which can come before the expiration date of the option $T$. For all securities considered herein, the associated reward function $f(t, x)$ is defined through $T$.

This problem can be represented in an alternative probabilistic form. To this end, we first define the process

$$
Y_{t}=e^{-r t} f\left(t, X_{t}\right), \quad 0 \leq t \leq T .
$$

By (4) and Ito's formula, we obtain the SDE

$$
\begin{aligned}
d Y_{t} & =e^{-r t}\left(-r f\left(t, X_{t}\right)+f_{t}\left(t, X_{t}\right)+f_{x}\left(t, X_{t}\right) A\left(t, X_{t}\right)+\frac{\sigma^{2}}{2} f_{x x}\left(t, X_{t}\right)\right) d t+e^{-r t} f_{x}\left(t, X_{t}\right) \sigma d W_{t} \\
& =e^{-r t} G\left(t, X_{t}\right) d t+e^{-r t} f_{x}\left(t, X_{t}\right) \sigma d W_{t}
\end{aligned}
$$

where we have denoted $f_{t} \equiv \frac{\partial f}{\partial t}, f_{x} \equiv \frac{\partial f}{\partial x}, f_{x x} \equiv \frac{\partial^{2} f}{\partial x^{2}}$, and

$$
G(t, x):=-r f(t, x)+f_{t}(t, x)+f_{x}(t, x) A(t, x)+\frac{\sigma^{2}}{2} f_{x x}(t, x) .
$$

The function $G(t, x)$, called the drive function (see Leung and Shirai (2015) for the terminology), determines the sign of the drift of the SDE for the discounted reward process $Y_{t}=e^{-r t} f\left(t, X_{t}\right)$.

Integrating (27) and substituting in (25), the value function can be expressed as

$$
V(t, x)=\sup _{\tau \in \mathcal{T}_{t, T}} \mathbb{E}\left\{\int_{t}^{\tau} e^{-r(u-t)} G\left(u, X_{u}\right) d u \mid X_{t}=x\right\}+f(t, x) .
$$

Rearranging the terms in (29), we define the difference between the value function $V(t, x)$ and the reward function $f(t, x)$ to be the delayed liquidation premium, i.e.,

$$
\begin{aligned}
L(t, x) & :=V(t, x)-f(t, x) \\
& =\sup _{\tau \in \mathcal{T}_{t, \bar{T}}} \mathbb{E}\left\{\int_{t}^{\tau} e^{-r(u-t)} G\left(u, X_{u}\right) d u \mid X_{t}=x\right\} .
\end{aligned}
$$

For every position held, there is an embedded timing option to sell. The delayed liquidation premium quantifies the value of optimally waiting to exercise this timing option. Since $V(t, x) \geq f(t, x)$ for all $(t, x)$, this follows from (30) that $L(t, x) \geq 0$ for all $(t, x)$, meaning that the delayed liquidation premium is always positive. 
By standard optimal stopping theory (Karatzas and Shreve 1998, Theorem D.12), the optimal liquidation time, associated with $V(t, x)$ or $L(t, x)$, is given by

$$
\begin{aligned}
\tau^{*} & =\inf \left\{u \in[t, \bar{T}]: V\left(u, X_{u}\right)=f\left(u, X_{u}\right)\right\} \\
& =\inf \left\{u \in[t, \bar{T}]: L\left(u, X_{u}\right)=0\right\}
\end{aligned}
$$

In other words, it is optimal for the trader to exercise the timing option and close out the position as soon as the optimal liquidation premium $L$ vanishes. Accordingly, the trader's optimal liquidation strategy can be described by the exercise region $\mathcal{S}$ and continuation region $\mathcal{D}$, namely,

$$
\begin{aligned}
& \mathcal{S}=\left\{\left(t, e^{x}\right) \in[0, \bar{T}] \times \mathbb{R}_{+}: L(t, x)=0\right\}, \\
& \mathcal{D}=\left\{\left(t, e^{x}\right) \in[0, \bar{T}] \times \mathbb{R}_{+}: L(t, x)>0\right\} .
\end{aligned}
$$

On the other hand, if the delayed liquidation premium is always strictly positive, then the trader finds it optimal to wait through the end of the trading horizon. In particular, we may have $L(\bar{T}, x)>0$ for all $x$, so we interpret $\tau^{*}=\bar{T}$ as never exercising at all. Therefore, we can now identify the conditions under which it is optimal to immediately liquidate, or hold the asset/option position through $\bar{T}$.

Proposition 1. Let $t \in[0, \bar{T}]$ be the current time. Then, we have

1. $G(u, x)>0, \forall(u, x) \in[t, \bar{T}] \times \mathbb{R}_{+} \Longrightarrow \tau^{*}=\bar{T}$,

2. $G(u, x) \leq 0, \forall(u, x) \in[t, \bar{T}] \times \mathbb{R}_{+} \Longrightarrow \tau^{*}=t$.

Proof. From (29), if $G(u, x)$ is positive (resp. negative) $\forall(u, x) \in[t, \bar{T}] \times \mathbb{R}^{+}$, then the discounted reward function is maximized at the longest (resp. shortest) stopping time, i.e., $\tau^{*}=\bar{T}$ (resp. $\left.\tau^{*}=t\right)$.

In addition to the two extremal cases, we can also address other cases and solve for the trader's nontrivial trading strategies. To do so, let us write down the variational inequality associated with the value function $V(t, x)$ (see (25)) with a general reward function $f(t, x)$. First, define the differential operator

$$
\mathcal{L}\{\cdot\}:=-r \cdot+\frac{\partial \cdot}{\partial t}+A(t, x) \frac{\partial \cdot}{\partial x}+\frac{\sigma^{2}}{2} \frac{\partial^{2}}{\partial x^{2}} .
$$

The optimal stopping problem $V$ is solved from the variational inequality:

$$
\max \{\mathcal{L} V(t, x), f(t, x)-V(t, x)\}=0,
$$

where $(t, x) \in[0, \bar{T}) \times \mathbb{R}$, with the terminal condition $V(\bar{T}, x)=f(\bar{T}, x)$ for all $x \in \mathbb{R}$. We refer to (Leung and Shirai 2015, sct. 7) for a detailed proof for the existence and uniqueness of a strong solution to variational inequalities of this form. A more comprehensive reference is Bensoussan and Lions (1982). We will discuss in Section 4 our numerical scheme to solve for the optimal trading strategies.

Next, we examine the trading strategies for stock and options, and study the varying effects of the trader's belief encoded in the random variable $D$. For each security type, we will derive the corresponding drive function. It will then be the inputs for the variational inequality (35), which will be solved numerically for the optimal trading strategies. We consider several combinations in securities and beliefs to see any major differences in the strategies. 


\subsection{Stocks}

For selling the stock $S$, the reward function is simply $f(x)=e^{x}$. Then, the drive function, denoted by $G_{s t o c k}(t, x)$, for selling the stock can be computed via (28). Precisely, we get

$$
G_{\text {stock }}(t, x)=\left(-r+A(t, x)+\frac{1}{2} \sigma^{2}\right) e^{x},
$$

for $(t, x) \in[0, T] \times \mathbb{R}$. As seen in Figure 2, the function $A(t, x)$ and thus drive function depend heavily on the prescribed distribution of $D$, and may be nonlinear. In general, it is difficult to pinpoint the behavior of the drive function $G_{\text {stock }}(t, x)$. Nevertheless, under the normal distribution for $D$, we obtain the following properties for $G_{\text {stock }}$ in $x$ and in $t$, respectively.

Proposition 2. Suppose that the trader's belief on log-price follows a normal distribution as in Example 2. Then, the drive function $G_{\text {stock }}(t, x)$ given in (36) is

(i) downward-sloping and concave in $x$ if

$$
\sigma_{D}>\sqrt{T} \sigma \text { and } x \leq q(t)-2
$$

or

$$
\sigma_{D}<\sqrt{T} \sigma \text { and } x \geq q(t)-1
$$

(ii) downward-sloping and convex in $x$ if

$$
\sigma_{D}>\sqrt{T} \sigma \text { and } q(t)-2 \leq x \leq q(t)-1,
$$

(iii) upward-sloping and concave in $x$ if

$$
\sigma_{D}<\sqrt{T} \sigma \text { and } q(t)-2 \leq x \leq q(t)-1,
$$

(iv) upward-sloping and convex in $x$ if

$$
\sigma_{D}>\sqrt{T} \sigma \text { and } x \geq q(t)-1,
$$

or

$$
\sigma_{D}<\sqrt{T} \sigma \text { and } x \leq q(t)-2
$$

where

$$
q(t):=X_{0}-\frac{\mu \sigma^{2} T+\left(\frac{1}{2} \sigma^{2}-r\right)\left(t \sigma_{D}^{2}+T \sigma^{2}(T-t)\right)}{\sigma_{D}^{2}-T \sigma^{2}} .
$$

Proposition 3. Suppose that the trader's belief on log-price follows a normal distribution as in Example 2. Then, the drive function in (36) is

(i) downward-sloping and concave in $t$ if

$$
\sigma_{D}<\sqrt{T} \sigma \text { and } x \geq X_{0}-\frac{\mu \sigma^{2} T}{\sigma_{D}^{2}-T \sigma^{2}},
$$

(ii) downward-sloping and convex in $t$ if

$$
\sigma_{D}>\sqrt{T} \sigma \text { and } x \geq X_{0}-\frac{\mu \sigma^{2} T}{\sigma_{D}^{2}-T \sigma^{2}},
$$


(iii) upward-sloping and concave in $t$ if

$$
\sigma_{D}>\sqrt{T} \sigma \text { and } x \leq X_{0}-\frac{\mu \sigma^{2} T}{\sigma_{D}^{2}-T \sigma^{2}}
$$

(iv) upward-sloping and convex in $t$ if

$$
\sigma_{D}<\sqrt{T} \sigma \text { and } x \leq X_{0}-\frac{\mu \sigma^{2} T}{\sigma_{D}^{2}-T \sigma^{2}}
$$

The proof is provided in Section 6.3. In addition, if $\sigma_{D}=\sqrt{T} \sigma$, then the drive function is independent of $t$. Under the normal distribution for $D, G_{\text {stock }}(t, x)$ is upward-slopping and convex if $r<\frac{\mu}{T}+\frac{1}{2} \sigma^{2}$, and downward-slopping and concave if $r>\frac{\mu}{T}+\frac{1}{2} \sigma^{2}$. However, for two-point discrete distribution and double exponential distribution, the properties of the drive function are not explicitly available, but the function can be numerically computed instantly using (36).

Figure 3 presents the optimal trading strategies for selling a stock with beliefs corresponding to the two-point discrete distribution, normal distribution and double exponential distribution. Typically, as seen in panels (a), (b) and (d), we observe a decreasing boundary over time. This means that the trader tends to sell the stock when the stock price is sufficiently high, but is willing to liquidate at a lower price as the trading deadline approaches. However, Figure $3 \mathrm{~b}$ shows that the optimal boundary is increasing in time under the two-point discrete distribution for $D$ (see Example 1). Note that, in Figure 3b, the trader has a more divergent belief in the sense that the terminal stock price will end up either very high or very low. Under our model, this suggests that the trader will hold the stock if the price is high since the trader believes the price will likely increase further, and the trader will sell it if the price is low because the price is believed to go even lower under the trader's belief. Figure $3 a, b$ represent the trader's two contrasting beliefs. The magnitude of the parameter $\delta$ guides the trader's reaction to new price information and ultimately the trading strategy. This highlights that the behavior of the boundary can be significantly changed by the choice of distribution of $D$ and associated parameters.

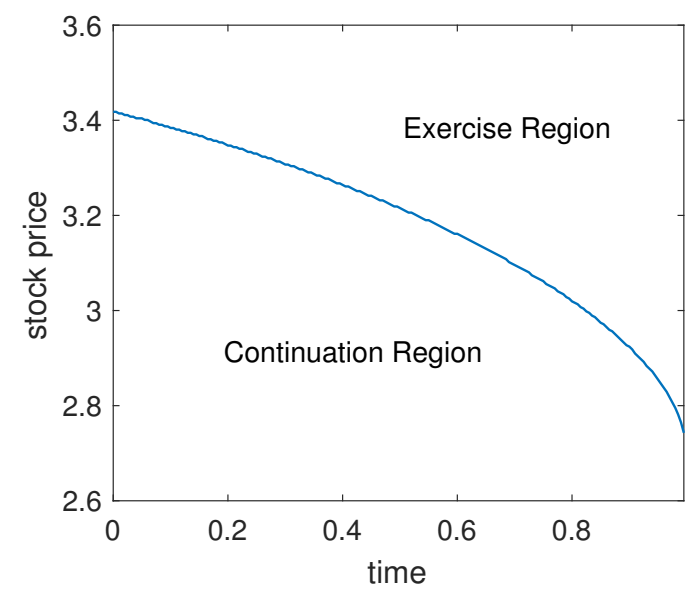

(a)

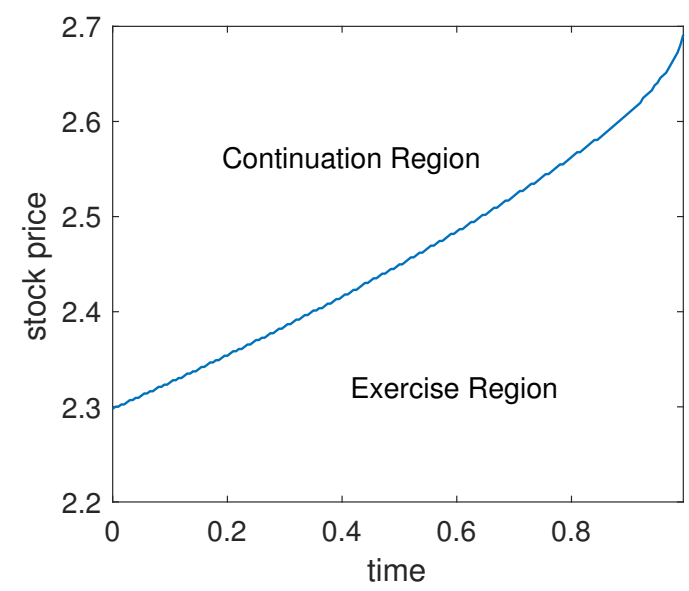

(b)

Figure 3. Cont. 


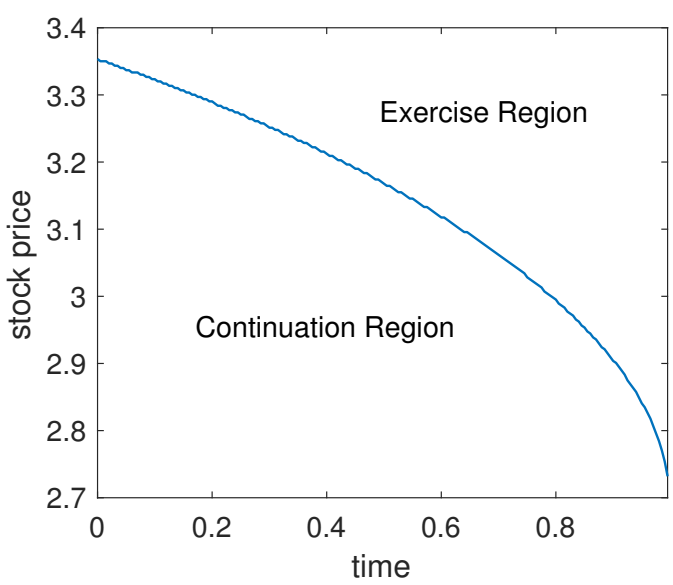

(c)

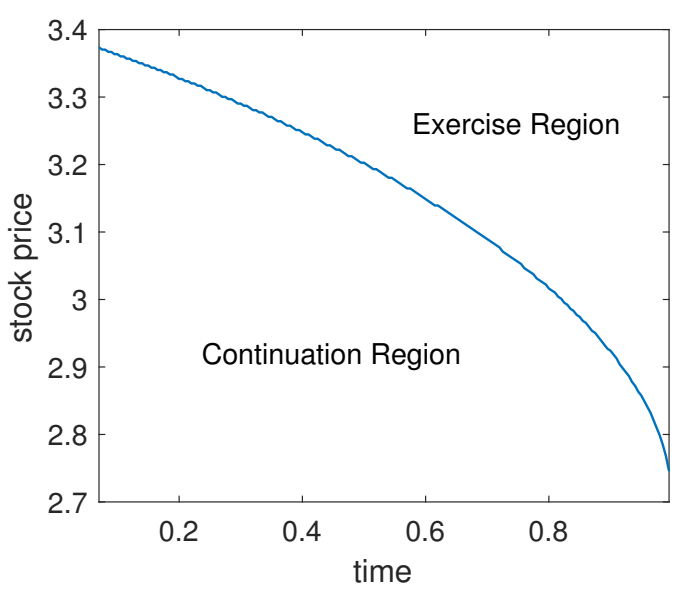

(d)

Figure 3. Optimal boundaries for selling stock under two-point discrete distribution (a,b), normal distribution (c) and double exponential distribution (d). Parameters for each plot are as follows: (a) $\delta_{u}=-\delta_{d}=0.1, p_{u}=p_{d}=0.5$; (b) $\delta_{u}=-\delta_{d}=2, p_{u}=p_{d}=0.5$; (c) $\mu=0, \sigma_{D}=0.2$; (d) $\theta=0$, $p_{1}=p_{2}=0.5, \lambda_{1}=\lambda_{2}=10$. Common parameters: $S_{0}=2.72\left(X_{0}=1\right), r=0.1, \bar{T}=1, T=1.1$, $\sigma=0.4$.

In Figure 4, we illustrate the sensitivities of the optimal strategies for selling stock with respect to a number of parameters under three different distributions for $D$. As expected, the boundary increases as the mean (left panels) of $D$ increases and variance decreases (right panels).

Due to the nonlinearity of the drive functions under certain distributions, the trader may need to adopt more complicated trading strategies. As we have shown in Figure 5, if the initial stock price is in the continuation region, then the trader does not sell until the price reaches the exercise boundaries. However, the continuation region is sandwiched by two upper and lower disconnected exercise regions. This means that not only when the price is sufficiently high but also sufficiently low, the trader will sell the stock. We also notice another continuation region when the stock price is very low. The intuition surrounding this continuation region is that when the current stock price is so low, the trader speculates that the price will rebound and chooses to hold onto the stock.

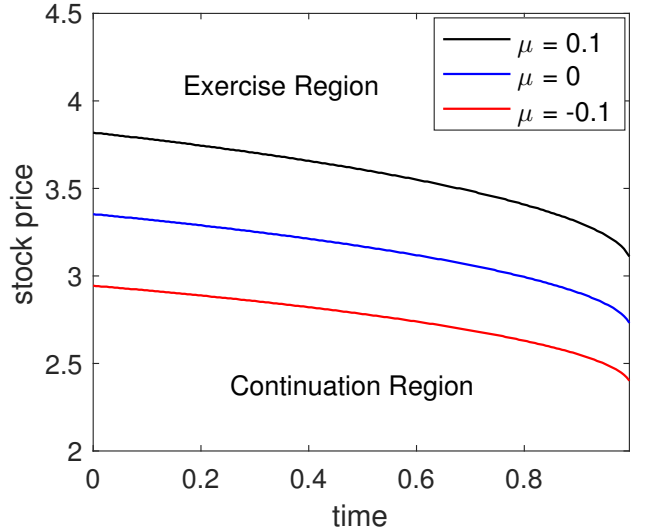

(a)

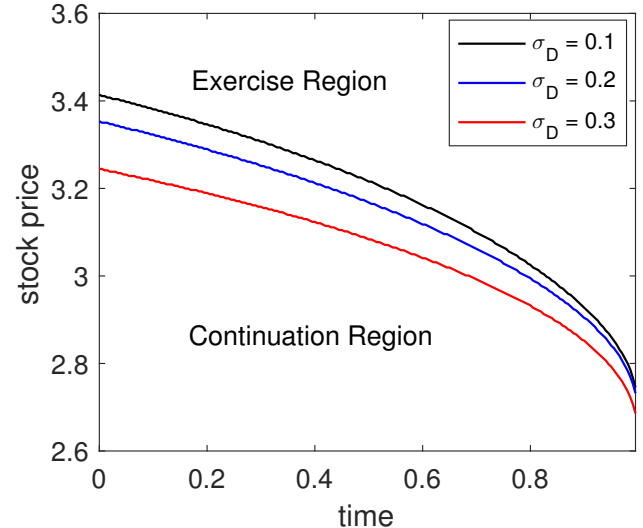

(b)

Figure 4. Cont. 


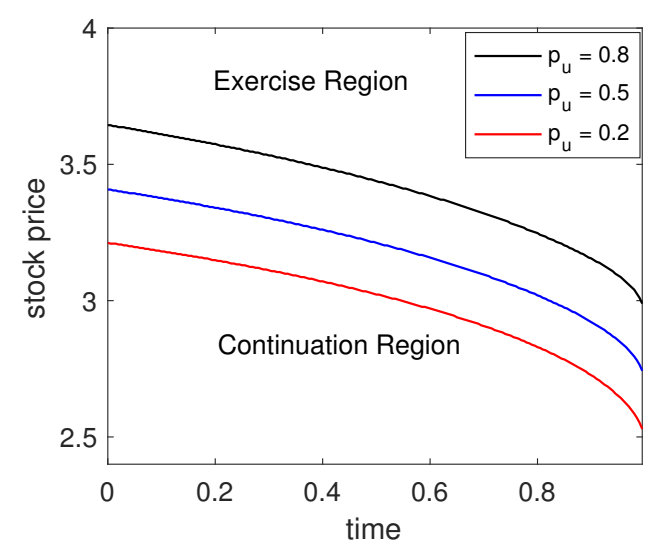

(c)

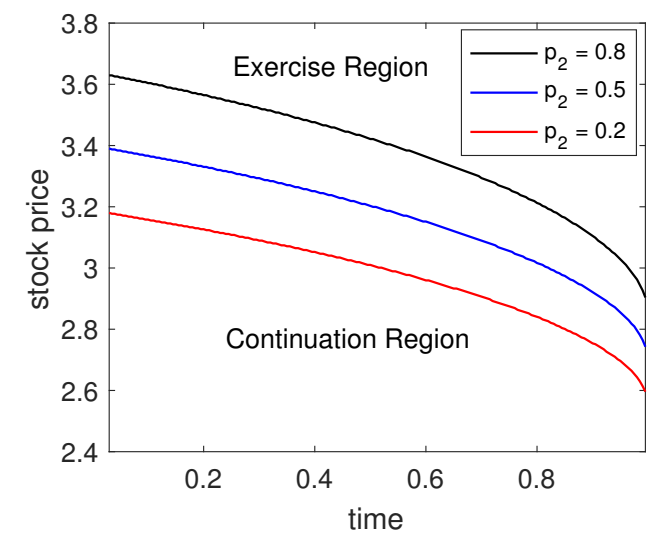

(e)

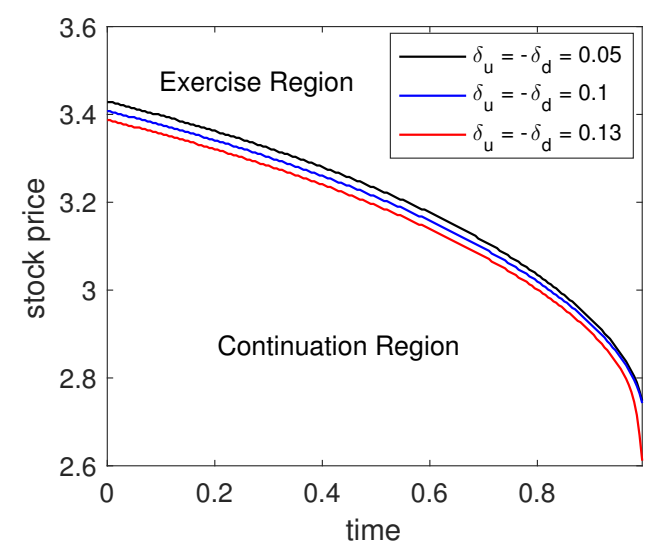

(d)

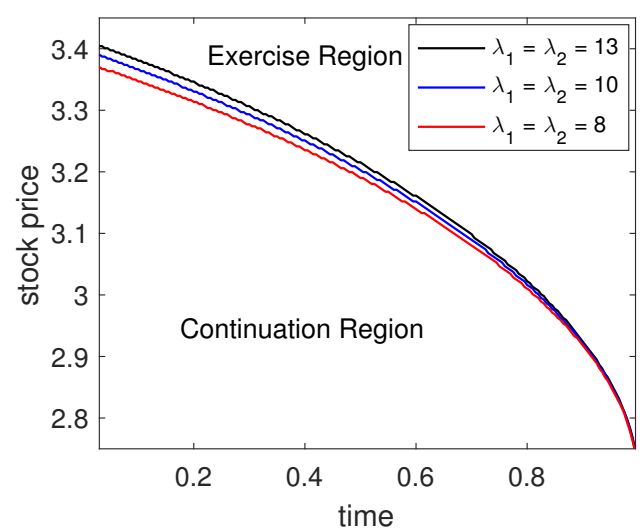

(f)

Figure 4. Optimal trading boundaries for stock liquidation under the two-point discrete distribution $(\mathbf{a}, \mathbf{b})$, normal distribution $(\mathbf{c}, \mathbf{d})$ and double exponential distribution $(\mathbf{e}, \mathbf{f})$. Parameters: $(\mathbf{a}) \sigma_{D}=0.2$; (b) $\mu=0$; (c) $\delta_{u}=-\delta_{d}=0.1$; (d) $p_{u}=p_{d}=0.5$; (e) $\lambda_{1}=\lambda_{2}=10$; (f) $p_{1}=p_{2}=0.5$. Other common parameters are the same as Figure 3.

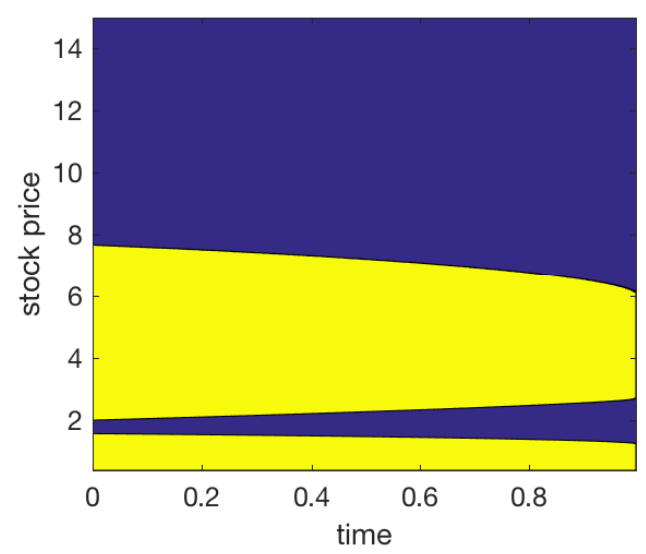

Figure 5. Optimal trading regions for stock liquidation under the two-point discrete distribution. The continuation regions are in yellow (light) color and the exercise regions are in blue (dark), and they are disconnected. Parameters: $\delta_{u}=-\delta_{d}=0.8, p_{u}=p_{d}=0.5$. Common parameters: $X_{0}=1, r=0.1$, $\bar{T}=1, T=1.1, \sigma=0.4$. 
Figure 6a suggests a different trading strategy under the double exponential distribution for $D$. Since the lower continuation region is relatively narrow, for most starting prices, the trader will find it optimal to sell the stock immediately. It is clear that trader's prior belief impacts her trading strategies. In Figure $6 \mathrm{~b}$, the parameters $\lambda_{1}$ and $\lambda_{2}$ are relatively small, and the mean and variance of $D$ are 0 and variance 0.16 (see (21) and (22)). Compared to the market view with the same mean but smaller variance of value 0.09 in Figure 6a, the upper continuation region is smaller (and vanishes for small $t$ ) and lower continuation region becomes larger in Figure $6 \mathrm{~b}$.

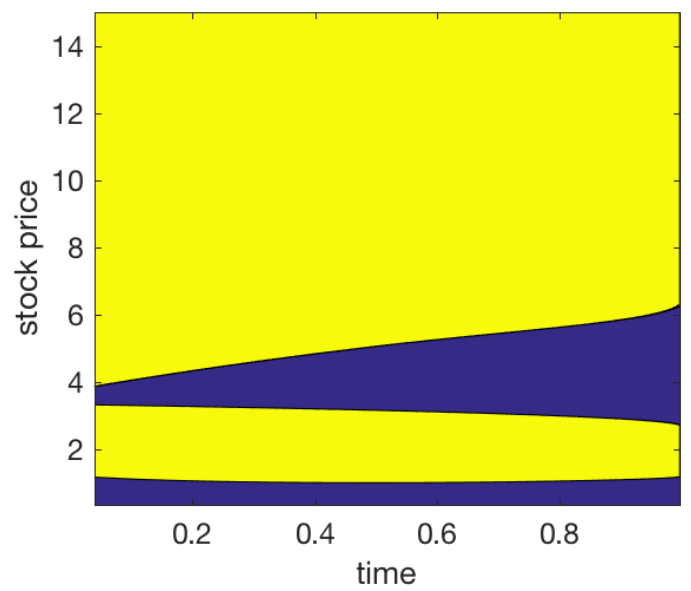

(a)

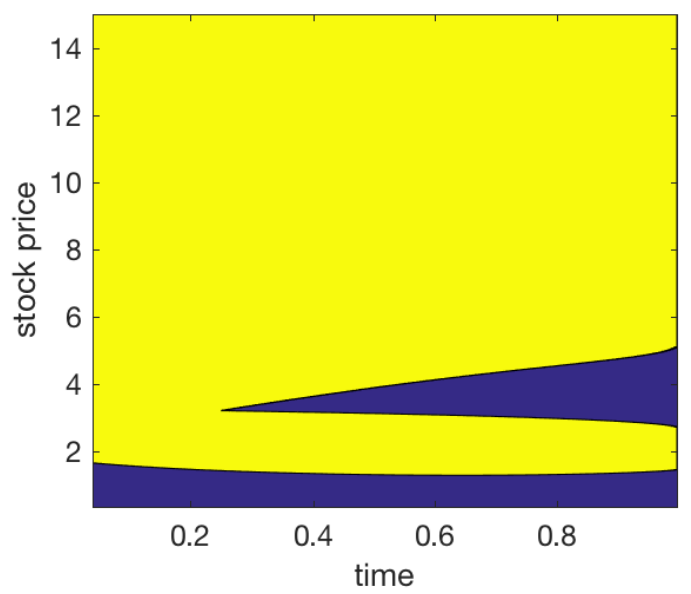

(b)

Figure 6. Optimal trading regions for stock liquidation under the double exponential distribution. The continuation regions are in yellow (light) color and the exercise regions are in blue (dark), and they are disconnected. Parameters for (a): $\theta=0, \lambda_{1}=\lambda_{2}=4.714, p_{1}=p_{2}=0.5$; parameters for $(\mathbf{b}): \theta=0, \lambda_{1}=\lambda_{2}=3.536, p_{1}=p_{2}=0.5$. Common parameters: $X_{0}=1, r=0.1$, $\bar{T}=1, T=1.1, \sigma=0.4$..

If the trader adopts the normal distribution for $D$ with mean zero and variance $\sigma^{2} T$, then she will never update the log-price dynamics because the drift of $X$ is zero, i.e., $A(t, x)=0 \forall(t, x)$. Other than this special degenerate case, the trader can take normal priors with different variances. We notice that the continuation/exercise regions are disconnected under the two-point discrete distribution and double exponential distribution in Figures 5 and 6, but there is a unique exercise boundary under normal distribution.

\subsection{Call and Put Options}

Given that the stock price follows SDE (4) under the historical measure $\mathbb{P}$, the risk-neutral counterpart follows the geometric Brownian motion

$$
d S_{t}=r S_{t} d t+\sigma S_{t} d W_{t}^{\mathbb{Q}},
$$

where $W_{t}^{\mathbb{Q}}$ is a standard Brownian motion under the risk-neutral measure $\mathbb{Q}$. Therefore, the no-arbitrage prices of European call and put options are found from the Black-Scholes pricing formulae.

The price of a European call with strike price $K$ and expiration date $T$ is given by

$$
C_{B S}\left(t, e^{x}\right)=\Phi\left(d_{1}\right) e^{x}-\Phi\left(d_{2}\right) K e^{-r(T-t)},
$$

for $(t, x) \in[0, T] \times \mathbb{R}$, where 


$$
\begin{aligned}
& d_{1}=\frac{1}{\sigma \sqrt{T-t}}\left(\ln \left(e^{x} / K\right)+\left(r+\sigma^{2} / 2\right)(T-t)\right), \\
& d_{2}=d_{1}-\sigma \sqrt{T-t} .
\end{aligned}
$$

Substituting the above into (28), we obtain the drive function

$$
\begin{aligned}
G_{\text {call }}(t, x) & =-r C_{B S}\left(t, e^{x}\right)+\frac{\partial C_{B S}\left(t, e^{x}\right)}{\partial t}+\frac{\partial C_{B S}\left(t, e^{x}\right)}{\partial x} A(t, x)+\frac{\sigma^{2}}{2} \frac{\partial^{2} C_{B S}\left(t, e^{x}\right)}{\partial x^{2}} \\
& =\left(-r+A(t, x)+\frac{\sigma^{2}}{2}\right) e^{x} \Phi\left(d_{1}\right),
\end{aligned}
$$

where $A(t, x)$ for the two-point, normal, double exponential cases are respectively computed by (14), (19) and (23).

In Figure 7a, we illustrate the optimal trading strategies for selling a European call under the normal distribution for $D$. As we can see, the trader tends to sell the call option when the stock price is high but is willing to sell at a lower price as time approaches maturity. The green (light) line shows where $G_{\text {call }}=0$, and the continuation region contains $G(t, x) \geq 0$. Recall that $G(t, x)$ is the integrand in the expression for $L(t, x)$. Naturally, if $G(t, x)>0$, it is optimal for the trader to continue to hold the call because she can obtain positive infinitesimal premium by waiting for an infinitesimally small amount of time. The same argument also applies to the cases with a stock and a put option.

Next, we consider the liquidation of a European put option. The Black-Scholes put price is given by

$$
P_{B S}\left(t, e^{x}\right)=\Phi\left(-d_{2}\right) K e^{-r(T-t)}-\Phi\left(-d_{1}\right) e^{x},
$$

where $K$ is the strike price, $T$ is the expiration date, and $d_{1}$ and $d_{2}$ are given by (39) and (40). Using (28), the corresponding drive function $G_{p u t}$ is

$$
G_{p u t}(t, x)=\left(r-A(t, x)-\frac{\sigma^{2}}{2}\right) e^{x} \Phi\left(-d_{1}\right)
$$

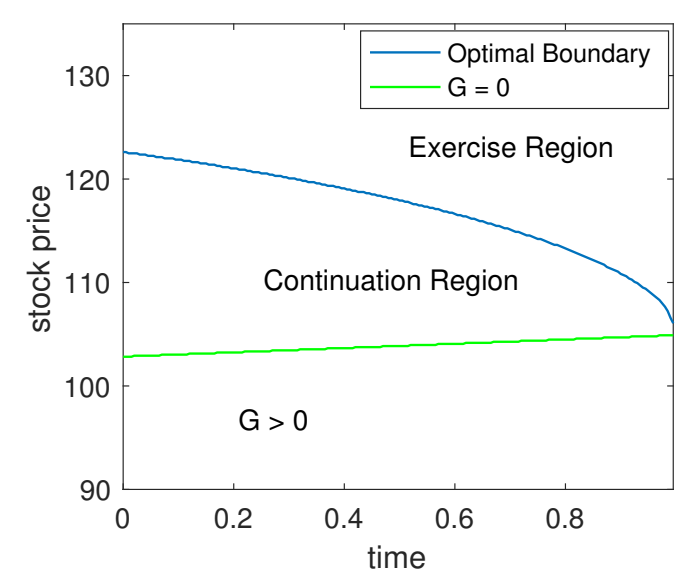

(a)

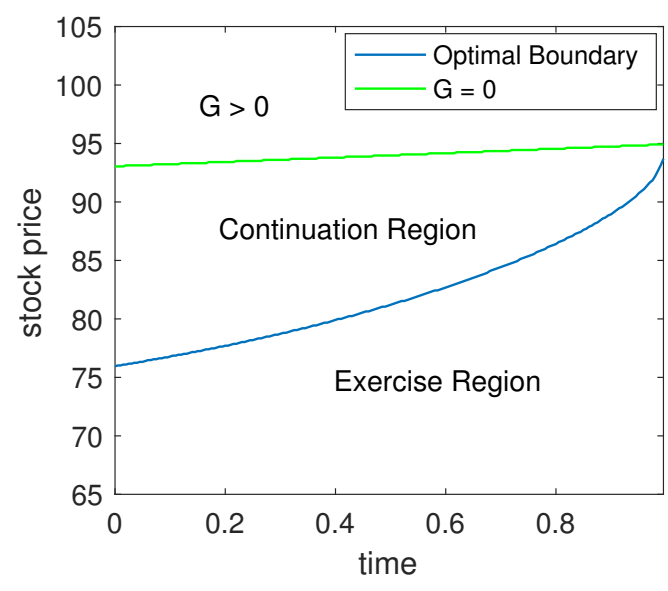

(b)

Figure 7. Optimal boundaries for selling European call with $S_{0}=105$ (a) and put with $S_{0}=95$ (b) under normal distribution. The strike price $K=100$ and maturity $T=1$, and the other parameters: $\mu=0, \sigma_{D}=0.1, r=0.1, \bar{T}=1, \sigma=0.4$. The green line is defined by $G=0$, and continuation region contains $G \geq 0$. 
Figure $7 \mathrm{~b}$ presents the optimal timing strategies for selling an European put under normal distribution. The figure shows that the trader tends to sell the put option when the stock price is low (i.e., when the put option price is high), but is willing to sell at a higher stock price (i.e., lower option price) as time approaches maturity. As a result, the exercise region expands and the continuation region shrinks as time progresses.

By direct calculation using the drive functions (41) and (43) for a call and a put, and comparing with the drive function (36) for the underlying asset, we arrive at the following parity result.

Lemma 1. (Call-Put Parity) Consider a pair of European call and put options with the same underlying $S$, strike $K$ and maturity T. Under model (4) with the same distribution for $D$, the associated drive functions satisfy

$$
G_{\text {call }}(t, x)-G_{\text {put }}(t, x)=G_{\text {stock }}(t, x),
$$

for all $(t, x) \in[0, T] \times \mathbb{R}$.

Proposition 4. Under the same distribution of D, consider European-style call and put options with the same strike and maturity. The optimal strategy to liquidate a long-call-short-put position is identical to the optimal strategy to sell the underlying stock under the same trading horizon.

Proof. The delayed liquidation premium associated with the long-call-short-put position is given by

$$
\begin{aligned}
L_{\text {call }- \text { put }}(t, x) & =\sup _{\tau \in \mathcal{T}_{t, T}} \mathbb{E}\left\{\int_{t}^{\tau} e^{-r(u-t)}\left(G_{\text {call }}\left(u, X_{u}\right)-G_{p u t}\left(u, X_{u}\right)\right) d u \mid X_{t}=x\right\} \\
& =\sup _{\tau \in \mathcal{T}_{t, \bar{T}}} \mathbb{E}\left\{\int_{t}^{\tau} e^{-r(u-t)} G_{\text {stock }}\left(u, X_{u}\right) d u \mid X_{t}=x\right\} .
\end{aligned}
$$

The last term is delayed liquidation premium associated with selling the stock $S$.

As an interesting consequence of Proposition 4, consider a pair of call and put with strike $K_{1}$ with maturity $T_{1}$, and another pair with strike $K_{2}$ and maturity $T_{2}$, with $\bar{T} \leq \min \left\{T_{1}, T_{2}\right\}$. The respective long-call-short-put positions will yield the same optimal timing strategy. This strategy is identical to that of selling the underlying stock $S$, which is independent of strike and maturity.

\section{Numerical Implementation}

We now summarize the numerical scheme used to solve the variational inequality satisfied by the value function for the optimal trading problem (25). A finite difference scheme is used to solve for the optimal liquidation boundaries.

The numerical solution of the system of variational inequalities can be obtained by applying a finite-difference scheme with the use of the Projected-Successive-Over-Relaxation (PSOR) method. The solution of the resulting equations for value functions are solved by the Successive Over Relaxation (SOR) method. We refer to Wilmott et al. (1995, chp. 9) for a detailed discussion on the projected SOR method. In each SOR iterative step in finding the numerical approximation of the value functions, we simply take the maximum value between the approximated function value and asset payoff. Then, the variational inequality (35) admits the linear complimentarity form:

$$
\left\{\begin{aligned}
\mathcal{L} V(t, x) \leq 0, \quad V(t, x) & \geq f(t, x), \quad(t, x) \in[0, \bar{T}) \times \mathbb{R}_{+}, \\
(\mathcal{L} V(t, x))(f(t, x)-V(t, x)) & =0, \quad(t, x) \in[0, \bar{T}) \times \mathbb{R}_{+}, \\
V(\bar{T}, x) & =f(\bar{T}, x), \quad x \in \mathbb{R}_{+} .
\end{aligned}\right.
$$


We now consider the discretization of the partial differential equation $\mathcal{L} V(t, x)=0$, over a bounded uniform grid with discretizations in time $(\delta t=\bar{T} / N)$, and space $\left(\delta x=\left(X_{\max }-X_{\min }\right) / M\right)$, where $X_{\max }$ and $X_{\min }$ are the upper and lower bounds on the values of $x$ on the grid. Applying the Crank-Nicolson method for $x$-derivatives and the backward difference for $t$-derivatives on the resulting equation leads to the grid equation:

$$
-\alpha_{i, j-1} V_{i-1, j-1}+(1-\beta) V_{i, j-1}-\gamma_{i, j-1} V_{i+1, j-1}=\alpha_{i, j} V_{i-1, j}+(1+\beta) V_{i, j}+\gamma_{i, j} V_{i+1, j}
$$

where the coefficients are given by

$$
\left\{\begin{aligned}
\alpha_{i, j} & =\delta t\left(\frac{\sigma^{2}}{4(\delta x)^{2}}-\frac{A\left(t_{j}, x_{i-1}\right)+A\left(t_{j}, x_{i+1}\right)}{8 \delta x}\right), \\
\beta & =-\frac{\delta t}{2}\left(r+\frac{\sigma^{2}}{(\delta x)^{2}}\right) \\
\gamma_{i, j} & =\delta t\left(\frac{\sigma^{2}}{4(\delta x)^{2}}+\frac{A\left(t_{j}, x_{i-1}\right)+A\left(t_{j}, x_{i+1}\right)}{8 \delta x}\right)
\end{aligned}\right.
$$

for $i=1,2, \ldots, M-1$ and $j=1,2, \ldots, N-1$. The system to be solved backward in time is

$$
\mathbf{M}_{\mathbf{j}-1}^{1} \mathbf{V}_{\mathbf{j}-1}=\mathbf{r}_{\mathbf{j}}
$$

where the right-hand side is

$$
\mathbf{r}_{\mathbf{j}}=\mathbf{M}_{\mathbf{j}}^{\mathbf{2}} \mathbf{V}_{\mathbf{j}}+\left[\begin{array}{c}
\alpha_{1, j-1} V_{0, j-1}+\alpha_{1, j} V_{0, j} \\
0 \\
\vdots \\
0
\end{array}\right]+\left[\begin{array}{c}
0 \\
\vdots \\
0 \\
\gamma_{M-1, j-1} V_{M, j-1}+\gamma_{M-1, j} V_{M, j}
\end{array}\right],
$$

and

$$
\begin{aligned}
& \mathbf{M}_{\mathbf{j}}^{1}=\left[\begin{array}{cccccc}
1-\beta & -\gamma_{1, j} & & & & \\
-\alpha_{2, j} & 1-\beta & -\gamma_{2, j} & & & \\
& -\alpha_{3, j} & 1-\beta & -\gamma_{3, j} & & \\
& & \ddots & \ddots & \ddots & \\
& & & -\alpha_{M-2, j} & 1-\beta & -\gamma_{M-2, j} \\
& & & & -\alpha_{M-1, j} & 1-\beta
\end{array}\right], \\
& \mathbf{M}_{\mathbf{j}}^{\mathbf{2}}=\left[\begin{array}{cccccc}
1+\beta & \gamma_{1, j} & & & & \\
\alpha_{2, j} & 1+\beta & \gamma_{2, j} & & & \\
& \alpha_{3, j} & 1+\beta & \gamma_{3, j} & & \\
& & \ddots & \ddots & \ddots & \\
& & & \alpha_{M-2, j} & 1+\beta & \gamma_{M-2, j} \\
& & & & \alpha_{M-1, j} & 1+\beta
\end{array}\right] \\
& \mathbf{V}_{\mathbf{j}}=\left[V_{1, j}, V_{2, j}, \ldots, V_{M-1, j}\right]^{T} .
\end{aligned}
$$


This leads to a sequence of stationary complementarity problems. Since the trader can close her position anytime prior to expiry, the value function $V(t, x)$ must satisfy the constraint

$$
V(t, x) \geq f(t, x), \quad X_{\min } \leq x \leq X_{\max }, \quad 0 \leq t \leq \bar{T} .
$$

Correspondingly, the discrete scheme can be written as

$$
V_{i, j} \geq f_{i, j}, \quad 0 \leq i \leq M, \quad 0 \leq j \leq N .
$$

Hence, at each time step $j \in\{1,2, \ldots, N-1\}$, we need to solve the linear complementarity problem

$$
\left\{\begin{aligned}
\mathbf{M}_{\mathbf{j}-\mathbf{1}}^{\mathbf{1}} \mathbf{V}_{\mathbf{j}-\mathbf{1}} & \geq \mathbf{r}_{\mathbf{j}} \\
\mathbf{V}_{\mathbf{j}-\mathbf{1}} & \geq \mathbf{f}_{\mathbf{j}-\mathbf{1}} \\
\left(\mathbf{M}_{\mathbf{j}-\mathbf{1}}^{\mathbf{1}} \mathbf{g}_{\mathbf{j}-\mathbf{1}}-\mathbf{r}_{\mathbf{j}}\right)^{T}\left(\mathbf{f}_{\mathbf{j}-\mathbf{1}}-\mathbf{g}_{\mathbf{j}-\mathbf{1}}\right) & =0
\end{aligned}\right.
$$

In particular, our algorithm enforces the constraint of dominating the payoff function as follows:

$$
V_{i, j-1}^{\text {new }}=\max \left\{V_{i, j-1}^{\text {old }}, f_{i, j-1}\right\}
$$

The projected SOR method is used to solve the linear system. Notice that the constraint is enforced at the same time as $V_{i, j-1}^{(k+1)}$ is calculated in each iteration. Hence, at each time step $j$, the PSOR algorithm is to iterate (on $k$ ) the equations

$$
\begin{aligned}
V_{1, j-1}^{(k+1)} & =\max \left\{f_{1, j-1}, V_{1, j-1}^{(k)}+\frac{\omega}{1-\beta}\left[r_{1, j}-(1-\beta) V_{1, j-1}^{(k)}+\gamma_{1, j-1} V_{2, j-1}^{(k)}\right]\right\}, \\
V_{2, j-1}^{(k+1)} & =\max \left\{f_{2, j-1}, V_{2, j-1}^{(k)}+\frac{\omega}{1-\beta}\left[r_{2, j}+\alpha_{2, j-1} V_{1, j-1}^{(k+1)}-(1-\beta) V_{2, j-1}^{(k)}+\gamma_{2, j-1} V_{3, j-1}^{(k)}\right]\right\}, \\
\vdots & \\
V_{M-1, j-1}^{(k+1)} & =\max \left\{f_{M-1, j-1}, V_{M-1, j-1}^{(k)}+\frac{\omega}{1-\beta}\left[r_{M-1, j}+\alpha_{M-1, j-1} V_{M-2, j-1}^{(k+1)}-(1-\beta) V_{M-1, j-1}^{(k)}\right]\right\},
\end{aligned}
$$

where $k$ is the iteration counter and $\omega$ is the overrelaxation parameter. The iterative scheme starts from an initial point $\mathbf{V}_{\mathbf{j}-\mathbf{1}}^{(0)}$ and proceeds until a convergence criterion is met, such as $\left\|\mathbf{V}_{\mathbf{j}-\mathbf{1}}^{(k+1)}-\mathbf{V}_{\mathbf{j}-\mathbf{1}}^{(k)}\right\|<\epsilon$, where $\epsilon$ is a tolerance parameter. The optimal boundary is identified by locating the grid points separating the exercise (or sell) region and continuation region, respectively defined by $\{(t, x)$ : $V(t, x)=f(t, x)\}$ and $\{(t, x): V(t, x)>f(t, x)\}$.

\section{Conclusions}

We have studied the optimal timing to sell an asset or option when the underlying asset's log-price follows a randomized Brownian bridge. By incorporating the trader's prior belief on the terminal stock price and allowing it to be updated with new price information, this approach can shed light on the effect of belief on the optimal trading strategy. We have explicitly derived the price dynamics under different beliefs (e.g., two-point discrete, double exponential, and normal distributions), and analyzed the properties of the associated delayed liquidation premia via the drive functions. By numerically solving the underlying variational inequality, we obtain the optimal trading strategies expressed in terms of sell/hold boundaries or regions. In particular, we find that the optimal strategy for liquidating a stock can admit connected or disconnected continuation/exercise regions under the two-point discrete distribution or double exponential distribution depending on the parameters' values. 
For future research, a natural direction is to consider under the current stochastic framework more sophisticated timing strategies for options or other derivatives, such as futures (see Leung et al. (2016)) and credit derivatives (see Leung and Liu (2012)). Other extensions include introducing additional stochastic factors that incorporate stochastic volatility and jumps to the Brownian bridge, and/or considering alternative distributions for the trader's prior belief.

\section{Proofs}

In this section, we provide the derivations for $a(t, x)$ given in Examples 2 (normal distribution) and 3 (double exponential distribution), respectively, in Sections 6.1 and 6.2. In addition, we provide the details for Propositions 2 and 3.

\subsection{Normal Distribution}

If the trader's prior belief on the log-price follows a normal distribution, then (6) can be expressed as

$$
a(t, x)=\frac{I_{1}(t, x)}{I_{2}(t, x)}
$$

where

$$
\left\{\begin{array}{l}
I_{1}(t, x)=\int_{-\infty}^{\infty} z \exp \left(z \frac{x-X_{0}}{\sigma^{2}(T-t)}-z^{2} \frac{t}{2 T \sigma^{2}(T-t)}-\frac{(z-\mu)^{2}}{2 \sigma_{D}^{2}}\right) d z, \\
I_{2}(t, x)=\int_{-\infty}^{\infty} \exp \left(z \frac{x-X_{0}}{\sigma^{2}(T-t)}-z^{2} \frac{t}{2 T \sigma^{2}(T-t)}-\frac{(z-\mu)^{2}}{2 \sigma_{D}^{2}}\right) d z .
\end{array}\right.
$$

Next, we compute $I_{1}$ and $I_{2}$ explicitly. To facilitate the representation, we denote

$$
\left\{\begin{array}{l}
\eta \equiv \eta(t)=\frac{t}{2 T \sigma^{2}(T-t)}+\frac{1}{2 \sigma_{D}^{2}} \\
b \equiv b(t, x)=-\left(\frac{x-X_{0}}{\sigma^{2}(T-t)}+\frac{\mu}{\sigma_{D}^{2}}\right) \\
c=\frac{\mu^{2}}{2 \sigma_{D}^{2}} .
\end{array}\right.
$$

Substituting the above notations into $I_{1}(t, x)$ and $I_{2}(t, x)$ and rearranging terms, we have

$$
I_{2}(t, x)=\int_{-\infty}^{\infty} \exp \left(-\left(\eta z^{2}+b z+c\right)\right) d z
$$

By applying a change of variables $y=\eta\left(z+\frac{b}{2 \eta}\right)^{2}$, we obtain

$$
\begin{aligned}
I_{1}(t, x) & =\int_{-\infty}^{\infty} z \exp \left(-\left(\eta z^{2}+b z+c\right)\right) d z \\
& =\int_{\infty}^{0} \frac{1}{2 \eta} \exp \left(\frac{b^{2}-4 \eta c}{4 \eta}\right) \exp (-y) d y+\int_{0}^{\infty} \frac{1}{2 \eta} \exp \left(\frac{b^{2}-4 \eta c}{4 \eta}\right) \exp (-y) d y-\frac{b}{2 \eta} I_{2}(t, x) \\
& =-\frac{b}{2 \eta} I_{2}(t, x)
\end{aligned}
$$


It follows immediately that

$$
a(t, x)=\frac{I_{1}(t, x)}{I_{2}(t, x)}=-\frac{b}{2 \eta}=T \frac{\left(x-X_{0}\right) \sigma_{D}^{2}+\mu \sigma^{2}(T-t)}{t \sigma_{D}^{2}+T \sigma^{2}(T-t)} .
$$

This, together with (5), gives us

$$
\begin{aligned}
A\left(t, X_{t}\right) & =\frac{a\left(t, X_{t}\right)-\left(X_{t}-X_{0}\right)}{T-t} \\
& =\frac{\sigma_{D}^{2}\left(X_{t}-X_{0}\right)+T \sigma^{2}\left(\mu-\left(X_{t}-X_{0}\right)\right)}{t \sigma_{D}^{2}+T \sigma^{2}(T-t)} .
\end{aligned}
$$

\subsection{Double Exponential Distribution}

If the trader's prior belief on the log-price follows a double exponential distribution, then (6) can be expressed as

$$
a(t, x)=\frac{N_{1}(t, x)+N_{2}(t, x)}{H_{1}(t, x)+H_{2}(t, x)}
$$

where

$$
\left\{\begin{array}{l}
N_{1}(t, x)=\int_{-\infty}^{\theta} p_{1} \lambda_{1} z \exp \left(z \frac{x-X_{0}}{\sigma^{2}(T-t)}-z^{2} \frac{t}{2 T \sigma^{2}(T-t)}-\lambda_{1}(\theta-z)\right) d z, \\
N_{2}(t, x)=\int_{\theta}^{\infty} p_{2} \lambda_{2} z \exp \left(z \frac{x-X_{0}}{\sigma^{2}(T-t)}-z^{2} \frac{t}{2 T \sigma^{2}(T-t)}-\lambda_{2}(z-\theta)\right) d z, \\
H_{1}(t, x)=\int_{-\infty}^{\theta} p_{1} \lambda_{1} \exp \left(z \frac{x-X_{0}}{\sigma^{2}(T-t)}-z^{2} \frac{t}{2 T \sigma^{2}(T-t)}-\lambda_{1}(\theta-z)\right) d z \\
H_{2}(t, x)=\int_{\theta}^{\infty} p_{2} \lambda_{2} \exp \left(z \frac{x-X_{0}}{\sigma^{2}(T-t)}-z^{2} \frac{t}{2 T \sigma^{2}(T-t)}-\lambda_{2}(z-\theta)\right) d z .
\end{array}\right.
$$

Now, we compute $N_{1}(t, x), N_{2}(t, x), H_{1}(t, x)$ and $H_{2}(t, x)$ explicitly. By completing the squares with a change of variables $y=\sqrt{2 \zeta}\left(z+\frac{b_{1}}{2 \zeta}\right)$, we have

$$
\begin{aligned}
H_{1}(t, x) & =\int_{-\infty}^{\theta} p_{1} \lambda_{1} \exp \left(-\left(\zeta z^{2}+b_{1} z+c_{1}\right)\right) d z \\
& =p_{1} \lambda_{1} \frac{\sqrt{2 \pi}}{\sqrt{2 \zeta}} \exp \left(\frac{b_{1}^{2}-4 \zeta c_{1}}{4 \zeta}\right) \frac{1}{\sqrt{2 \pi}} \int_{-\infty}^{\sqrt{2 \zeta}\left(\theta+\frac{b_{1}}{2 \zeta}\right)} \exp \left(-\frac{y^{2}}{2}\right) d y \\
& =p_{1} \lambda_{1} \frac{\sqrt{\pi}}{\sqrt{\zeta}} \exp \left(\frac{b_{1}^{2}-4 \zeta c_{1}}{4 \zeta}\right) \Phi\left(d_{1}\right),
\end{aligned}
$$


where

$$
\left\{\begin{array}{l}
\zeta \equiv \zeta(t)=\frac{t}{2 T \sigma^{2}(T-t)^{\prime}} \\
b_{1} \equiv b_{1}(t, x)=-\left(\frac{x-X_{0}}{\sigma^{2}(T-t)}+\lambda_{1}\right) \\
c_{1}=\lambda_{1} \theta \\
d_{1} \equiv d_{1}(t, x)=\sqrt{2 \zeta(t)}\left(\theta+\frac{b_{1}(t, x)}{2 \zeta(t)}\right)
\end{array}\right.
$$

Similarly, by applying $y=\sqrt{2 \zeta}\left(z+\frac{b_{2}}{2 \zeta}\right)$, we obtain

$$
\begin{aligned}
H_{2}(t, x) & =\int_{\theta}^{\infty} p_{2} \lambda_{2} \exp \left(-\left(\zeta z^{2}+b_{2} z+c_{2}\right)\right) d z \\
& =p_{2} \lambda_{2} \frac{\sqrt{2 \pi}}{\sqrt{2 \zeta}} \exp \left(\frac{b_{2}^{2}-4 \zeta c_{2}}{4 \zeta}\right) \frac{1}{\sqrt{2 \pi}} \int_{\sqrt{2 \zeta}\left(\theta+\frac{b_{2}}{2 \zeta}\right)}^{\infty} \exp \left(-\frac{y^{2}}{2}\right) d y \\
& =p_{2} \lambda_{2} \frac{\sqrt{\pi}}{\sqrt{\zeta}} \exp \left(\frac{b_{2}^{2}-4 \zeta c_{2}}{4 \zeta}\right) \Phi\left(-d_{2}\right),
\end{aligned}
$$

where

$$
\left\{\begin{array}{l}
b_{2} \equiv b_{2}(t, x)=-\left(\frac{x-X_{0}}{\sigma^{2}(T-t)}-\lambda_{2}\right), \\
c_{2}=-\lambda_{2} \theta \\
d_{2} \equiv d_{2}(t, x)=\sqrt{2 \zeta(t)}\left(\theta+\frac{b_{2}(t, x)}{2 \zeta(t)}\right) .
\end{array}\right.
$$

Next, we compute $N_{1}$ and $N_{2}$ in the numerator with changes of variables $y=\zeta\left(z+\frac{b_{1}}{2 \zeta}\right)^{2}$ and $y=\zeta\left(z+\frac{b_{2}}{2 \zeta}\right)^{2}$, respectively:

$$
\begin{aligned}
N_{1}(t, x) & =\int_{-\infty}^{\theta} p_{1} \lambda_{1} z \exp \left(-\left(\zeta z^{2}+b_{1} z+c_{1}\right)\right) d z \\
& =-\int_{\zeta\left(\theta+\frac{b_{1}}{2 \zeta}\right)^{2}}^{\infty} p_{1} \lambda_{1} \frac{1}{2 \zeta} \exp \left(\frac{b_{1}^{2}-4 \zeta c_{1}}{4 \zeta}\right) \exp (-y) d y-\frac{b_{1}}{2 \zeta} H_{1}(t, x) \\
& =-p_{1} \lambda_{1} \frac{1}{2 \zeta} \exp \left(-\zeta\left(\theta+\frac{b_{1}}{2 \zeta}\right)^{2}\right) \exp \left(\frac{b_{1}^{2}-4 \zeta c_{1}}{4 \zeta}\right)-p_{1} \lambda_{1} \frac{b_{1}}{2 \zeta} \frac{\sqrt{\pi}}{\sqrt{\zeta}} \exp \left(\frac{b_{1}^{2}-4 \zeta c_{1}}{4 \zeta}\right) \Phi\left(d_{1}\right)
\end{aligned}
$$

and

$$
\begin{aligned}
N_{2}(t, x) & =\int_{\theta}^{\infty} p_{2} \lambda_{2} z \exp \left(-\left(\zeta z^{2}+b_{2} z+c_{2}\right)\right) d z \\
& =\int_{\zeta\left(\theta+\frac{b_{2}}{2 \zeta}\right)^{2}}^{\infty} p_{2} \lambda_{2} \frac{1}{2 \zeta} \exp \left(\frac{b_{2}^{2}-4 \zeta c_{2}}{4 \zeta}\right) \exp (-y) d y-\frac{b_{2}}{2 \zeta} H_{2}(t, x) \\
& =p_{2} \lambda_{2} \frac{1}{2 \zeta} \exp \left(-\zeta\left(\theta+\frac{b_{2}}{2 \zeta}\right)^{2}\right) \exp \left(\frac{b_{2}^{2}-4 \zeta c_{2}}{4 \zeta}\right)-p_{2} \lambda_{2} \frac{b_{2}}{2 \zeta} \frac{\sqrt{\pi}}{\sqrt{\zeta}} \exp \left(\frac{b_{2}^{2}-4 \zeta c_{2}}{4 \zeta}\right) \Phi\left(-d_{2}\right) .
\end{aligned}
$$




\subsection{Proof of Propositions 2 and 3}

Differentiating $G_{\text {stock }}(t, x)$ in (36) yields that

$$
\frac{\partial G_{\text {stock }}}{\partial x}(t, x)=\left(-r+\frac{1}{2} \sigma^{2}+\frac{\left(\sigma_{D}^{2}-T \sigma^{2}\right)\left(x-X_{0}\right)+\mu \sigma^{2} T+\sigma_{D}^{2}-T \sigma^{2}}{t \sigma_{D}^{2}+T \sigma^{2}(T-t)}\right) e^{x},
$$

and

$$
\frac{\partial^{2} G_{\text {stock }}}{\partial x^{2}}(t, x)=\left(-r+\frac{1}{2} \sigma^{2}+\frac{\left(\sigma_{D}^{2}-T \sigma^{2}\right)\left(x-X_{0}\right)+\mu \sigma^{2} T+2\left(\sigma_{D}^{2}-T \sigma^{2}\right)}{t \sigma_{D}^{2}+T \sigma^{2}(T-t)}\right) e^{x},
$$

and

$$
\frac{\partial G_{\text {stock }}}{\partial t}(t, x)=-\frac{\sigma_{D}^{2}-T \sigma^{2}}{t \sigma_{D}^{2}+T \sigma^{2}(T-t)} e^{x} A(t, x)
$$

and

$$
\frac{\partial^{2} G_{\text {stock }}}{\partial t^{2}}(t, x)=2\left(\frac{\sigma_{D}^{2}-T \sigma^{2}}{t \sigma_{D}^{2}+T \sigma^{2}(T-t)}\right)^{2} e^{x} A(t, x),
$$

where $A(t, x)$ is given by (19). The results follow directly from the signs of these partial derivatives. The proof of Proposition 3 is similar to Proposition 2.

Author Contributions: T.L. conceived and designed the study; T.L and J.L. developed the implementation methods and analyzed the results and data; T.L., J.L., and X.L. derived the analytical results and wrote the paper.

Funding: This research received no external funding.

Conflicts of Interest: The authors declare no conflict of interest.

\section{References}

Baurdoux, Erik J., Nan Chen, Budhi A. Surya, and Kazutoshi Yamazaki. 2015. Optimal double stopping of a Brownian bridge. Advances in Applied Probability 47: 1212-34. [CrossRef]

Bensoussan, Alain, and Jacques-Louis Lions. 1982. Applications of Variational Inequalities in Stochastic Control. Amsterdam: North-Holland Publishing Co.

Brennan, Michael J., and Eduardo S. Schwartz. 1990. Arbitrage in stock index futures. Journal of Business 63: S7-S31. [CrossRef]

Brody, Dorje C., Mark HA Davis, Robyn L. Friedman, and Lane P. Hughston. 2009. Informed traders. In Proceedings of the Royal Society of London A: Mathematical, Physical and Engineering Sciences. London: The Royal Society, vol. 465, pp. 1103-1122.

Brody, Dorje C., Lane P. Hughston, and Andrea Macrina. 2008b. Information-based asset pricing. International Journal of Theoretical and Applied Finance 11: 107-42. [CrossRef]

Cartea, Álvaro, Sebastian Jaimungal, and Damir Kinzebulatov. 2016. Algorithmic trading with learning. International Journal of Theoretical and Applied Finance 19: 1650028. [CrossRef]

Dai, Min, Yifei Zhong, and Yue Kuen Kwok. 2011. Optimal arbitrage strategies on stock index futures under position limits. Journal of Futures Markets 31: 394-406. [CrossRef]

Ekström, Erik, and Juozas Vaicenavicius. 2017. Optimal stopping of a Brownian bridge with an unknown pinning point. arXiv preprint arXiv:1705.00369.

Ekström, Erik, and Henrik Wanntorp. 2009. Optimal stopping of a Brownian bridge. Journal of Applied Probability 46: 170-80. [CrossRef]

Filipović, Damir, Lane P. Hughston, and Andrea Macrina. 2012. Conditional density models for asset pricing. International Journal of Theoretical and Applied Finance 15: 1250002. [CrossRef]

Guo, Kevin, and Tim Leung. 2017. Understanding the non-convergence of agricultural futures via stochastic storage costs and timing options. Journal of Commodity Markets 6: 32-49. [CrossRef] 
Hughston, Lane P., and Andrea Macrina. 2012. Pricing fixed-income securities in an information-based framework. Applied Mathematical Finance 19: 361-79. [CrossRef]

Johannes, Michael, and Andrew Dubinsky. 2006. Earnings Announcements and Equity Options. Unpublished Paper. New York: Columbia University.

Karatzas, Ioannis, and Steven E. Shreve. 1998. Methods of Mathematical Finance. New York: Springer.

Kou, Steven G. 2002. A jump-diffusion model for option pricing. Management Science 48: 1086-101. [CrossRef]

Leung, Tim, Jiao Li, Xin Li, and Zheng Wang. 2016. Speculative futures trading under mean reversion. Asia-Pacific Financial Markets 23: 281-304. [CrossRef]

Leung, Tim, and Peng Liu. 2012. Risk premia and optimal liquidation of credit derivatives. International Journal of Theoretical \& Applied Finance 15: 1250059.

Leung, Tim, and Mike Ludkovski. 2011. Optimal timing to purchase options. SIAM Journal on Financial Mathematics 2: 768-93. [CrossRef]

Leung, Tim, and Mike Ludkovski. 2012. Accounting for risk aversion in derivatives purchase timing. Mathematics $\mathcal{E}$ Financial Economics 6: 363-86.

Leung, Tim, and Marco Santoli. 2014. Accounting for earnings announcements in the pricing of equity options. Journal of Financial Engineering 1: 1450031. [CrossRef]

Leung, Tim, and Marco Santoli. 2016. Leveraged Exchange-Traded Funds: Price Dynamics and Options Valuation. SpringerBriefs in Quantitative Finance. New York: Springer.

Leung, Tim, and Yoshihiro Shirai. 2015. Optimal derivative liquidation timing under path-dependent risk penalties. Journal of Financial Engineering 2: 1550004. [CrossRef]

Macrina, Andrea. 2014. Heat kernel models for asset pricing. International Journal of Theoretical and Applied Finance 17: 1450048. [CrossRef]

Wilmott, Paul, Sam Howison, and Jeff Dewynne. 1995. The Mathematics of Financial Derivatives: A Student Introduction, 1st ed. Cambridge: Cambridge University Press.

(C) 2018 by the authors. Licensee MDPI, Basel, Switzerland. This article is an open access article distributed under the terms and conditions of the Creative Commons Attribution (CC BY) license (http:/ / creativecommons.org/licenses/by/4.0/). 